\title{
Los conocimientos mínimos de Geografía en los estudios de Grado en Educación Primaria
}

\section{The minimum knowledge of Geography in the Bachelor's degree in Primary Education}

\author{
Jaume BINIMELIS SEBASTIÁN y Antoni ORDINAS GARAU \\ Universitat de les illes Balears
}

Recibido: Marzo 2015

Evaluado: Mayo 2015

Aceptado: Junio 2015

\begin{abstract}
Resumen
La asignatura Ciencias Sociales y su Didáctica I (Geografía) de los estudios de Grado en Educación Primaria es, en la Universitat de les Illes Balears y en la mayoría de universidades españolas, el único contacto académico que mantendrán los futuros maestros con la Geografía. Existe un ambiente de preocupación sobre la formación de los maestros en España, inquietud que no es exclusiva de los geógrafos ni de nuestro país. El presente trabajo indaga en los conocimientos mínimos de Geografía exigibles a los futuros docentes a través de una prueba de conocimientos basada, en gran parte, en el currículo de Ciencias Sociales de Educación Primaria de la Comunidad Autónoma de las Islas Baleares. Los resultados obtenidos demuestran que el alumnado del Grado en Educación Primaria no ha interiorizado esos contenidos tras su dilatada trayectoria académica. Asimismo desvelan que aunque ninguna variable es determinante, el género, la escasa presencia de materias de Geografía en el sistema educativo español y el nivel de formación de los padres son algunos de los factores que inciden en los resultados alcanzados por los alumnos en una prueba piloto sobre la que se basa la investigación.
\end{abstract}

Palabras clave: formación del profesorado, conocimientos mínimos, Geografía, Islas Baleares.

\begin{abstract}
The subject Social Sciences and its Didactics I (Geography) taught in the Bachelor's degree in Primary Teacher Education is, at the University of Balearic Islands and in most Spanish universities, the only academic contact that future teachers will have with Geography. There is an atmosphere of concern for teacher training in Spain, a worry which is not exclusive of geographers or our country. This paper investigates the minimum knowledge of Geography required to future teachers through a knowledge test based, mainly, on the Social Sciences curriculum of Primary Education of the Autonomous Community of the Balearic Islands. The results obtained demonstrate that the students of Bachelor's degree in Primary Education have not learnt these contents after their long academic career. The results also reveal that even though there is no determinant variable, gender, the scarce presence of Geography subjects in the Spanish education system and the education level of the parents are some of the factors that influence the results achieved by students in a pilot test on which our research is based.
\end{abstract}

Keywords: teacher training, minimum knowledge, Geography, Balearic Islands. 
El plan de estudios del grado de Maestro de Educación Primaria sólo contempla una asignatura de Geografía. Es fácil comprobar en el currículo de Primaria, ESO y Bachillerato, la escasa presencia de contenidos cognitivos y procedimentales ligados al saber geográfico, mal crónico en el sistema educativo español. Desde esta óptica, destaca el informe de Isaac Buzo Sánchez y Paloma Ibarra Benlloch (2014: 10) sobre la situación de la Geografía en ESO y Bachillerato. A partir de una encuesta dirigida al profesorado, dan buena cuenta del escaso peso de la Geografía en ambas etapas y de las interrupciones en la enseñanza de la Geografía, con varios cursos sin opción alguna de continuidad.

En este contexto se justifica la necesidad de reflexionar sobre los conocimientos mínimos exigibles en Geografía y en la posibilidad de indagar en ese campo a través de una prueba de conocimientos previos basada en el currículo de Ciencias Sociales de la comunidad autónoma balear, con las modificaciones que en el mismo ha introducido la LOMCE (GIB, 2014). Previamente, idénticas inquietudes se apuntaban, de forma implícita, en el Libro Blanco del Título de Grado en Magisterio (ANECA, 2005). Este documento indica que, entre las competencias comunes de los diferentes perfiles de Maestro, el ítem más valorado de los académicos consultados para elaborar las competencias comunes a todos los perfiles del título de Maestro es el "Conocimiento de los contenidos que hay que enseñar, comprendiendo su singularidad epistemológica y la especificidad de su didáctica". En general, los contenidos cognitivos (saber) preceden a los procedimentales (saber hacer) y también a los actitudinales (saber estar, saber ser), razón por la cual se concluyó que se observa "... una concepción muy clásica y tradicional del maestro..." (ANECA, 2005: 92). En definitiva, el Libro Blanco del grado en Estudios de Magisterio recoge un abanico de competencias en el que, de forma genérica o específica, hay un claro interés por lo que sepan los futuros maestros. Ese es el objetivo que también nos ha guiado en la indagación sobre los conocimientos mínimos en Geografía del estudiantado de Magisterio en Educación Primaria.

Para comprobar la suficiencia de dichos conocimientos hemos desarrollado un trabajo de investigación, con una prueba piloto que tenía como finalidad última la certificación de unos conocimientos mínimos entre el alumnado de segundo curso del grado de Educación Primaria en el ámbito de la Geografía. El test indaga en el arraigo de los contenidos propios de la Geografía de acuerdo con el currículo de Educación Primaria, antes comentado.

Las pruebas de conocimientos mínimos tienen, entre otros objetivos, evaluar si los estudiantes, en este caso de Magisterio, poseen un nivel de conocimientos adecuados. Existe un ambiente de preocupación sobre la formación de los maestros en España, como se apunta en un reciente trabajo de Sureda (2014). Esta inquietud no es exclusiva de los geógrafos. El propio Sureda (2014: 69-72), en un análisis sobre la formación de los maestros de infantil y primaria, centrado en el caso de las islas Baleares, hace hincapié en los conocimientos iniciales de los futuros maestros y, en este sentido, menciona una prueba de conocimientos matemáticos realizada en el marco de la asignatura Matemáticas de Educación Primaria por los profesores Miró y Nadal (Miró, 
J.; Nadal, M., 2014). Dicha prueba, aplicada a 149 alumnos, sólo fue superada por un $37,6 \%$.

Existen, además, otros estudios donde se evidencian las deficiencias en la formación de los estudiantes de Magisterio en evaluaciones de conocimientos matemáticos. Es el caso, por ejemplo, de un análisis realizado por un equipo de la Universidad de la Laguna en relación a estudiantes de 7 universidades españolas (Socas, M.M., 2011). O más recientemente, en otro estudio donde se pretendía comprobar si los estudiantes de Magisterio poseían las competencias matemáticas correspondientes a sexto curso de Educación Primaria. Dicho trabajo se efectuó con una muestra de 309 estudiantes de la Universidad de Murcia (Nortes, R; Nortes, A, 2014).

En nuestro caso, la prueba piloto tiene como objetivo analizar cuál es el estado de los conocimientos en Geografia de los estudiantes de la asignatura Ciencias Sociales y su Didáctica I (Geografía).

\section{El currículo de Ciencias Sociales}

El Decreto 32/2014, de 18 de julio, establece el currículo de Educación Primaria en las Islas Baleares, en su adaptación a la Ley Orgánica de Mejora de la Calidad Educativa (Ley Orgánica 8/2013, de 9 de diciembre, BOE 295). Entre sus cambios, destaca la desaparición del área de Conocimiento del Medio Social, Natural y Cultural, y la división de la misma en dos áreas: Ciencias Naturales y Ciencias Sociales. Capel y Luis Urteaga (1986: 28-32) ya reivindicaron a mediados de los ochenta un currículo integrado de Ciencias Sociales para la Educación Primaria y el Bachillerato donde, además de conocimientos de Geografía e Historia, aparecieran otros contenidos propios de otras ramas de las Ciencias Sociales, como Antropología, Economía, Sociología, Psicología o Derecho. Han pasado tres décadas y la Geografía y la Historia tienen un papel todavía casi excluyente en Educación Primaria. Por otra parte, fueron bastantes las voces que criticaron la falta de ubicación del área de Conocimiento del Medio Social, Natural y Cultural (la materia que integraba los fundamentos de Geografía en Educación Primaria), tanto en su tratamiento sistemático (sistema social, sistema biológico, sistema físico-químico), como en su perspectiva patriótica. Fue tachada de listado de conocimientos enciclopédicos sin interrelación entre ellos y que sólo daban lugar a aprendizajes de tipo memorístico (Pérez Estévez, P.; Ramírez Martínez, S.; Xouto González, X., 1997).

El currículo de Ciencias Sociales de la Comunidad Autónoma de las Islas Baleares se divide en 4 bloques. El bloque 1 (Contenidos comunes) aborda contenidos procedimentales y actitudinales transversales que se deberán de asentar al abordar el proceso de enseñanza-aprendizaje del resto de bloques (Utilización de las tecnologías de la información y la comunicación para buscar y seleccionar información, simular procesos y presentar conclusiones, a modo de ejemplo). Del análisis del bloque 2 ( $E l$ mundo que nos rodea), se desprende que se presta una gran atención a la cartografía (el mapa, la representación gráfica de la Tierra, la escala, las coordenadas geográficas, etc.), al clima (tipos de tiempo, zonas climáticas, etc.) y al paisaje como eje vertebrador 
de la integración en el medio, en el territorio, de las variables del medio físico (relieve, clima, vegetación) y, por último, a las consecuencias de la acción humana sobre el medio (contaminación, cambio climático...) y a las soluciones a los desajustes que aquélla ha creado (desarrollo sostenible). En el bloque 3 (Vivir en sociedad), entre otros, aparecen los contenidos que abordan la Geodemografía (población, migraciones, etc.) y, en menor medida, las actividades económicas. Por último, el bloque 4 (Las huellas del pasado) está dedicado íntegramente a contenidos que forman parte en su mayoría del ámbito de estudio de la Historia. En definitiva, los contenidos pivotan (tabla 1) en torno al medio y el hombre, la Geografía concebida como una reflexión sobre la dialéctica hombre/medio (razón de más para incluir en el apartado 2 unos ítems dedicados a las consecuencias de la intervención humana sobre el medio, introduciendo el concepto de desarrollo sostenible).

\section{BLOQUES}

2. El mundo que nos rodea

\section{CONTENIDOS}

El Universo y el sistema solar: el Sol, los planetas. La representación de la Tierra. Orientación en el espacio. Cartografía: Planos y mapas, escalas. La atmósfera. Fenómenos atmosféricos. El tiempo atmosférico. El clima y factores climáticos. La hidrosfera. Distribución del agua en el planeta. La litosfera: características y tipos de roca. Paisajes naturales de las Islas Baleares. La diversidad geográfica de los paisajes de España: relieve e hidrografía. La diversidad geográfica de los paisajes de Europa: relieve, climas e hidrografía. La intervención humana en el medio. El desarrollo sostenible.

3. Vivir en sociedad

La organización social, política y territorial del Estado español. La Unión Europea. La población. Las actividades productivas. El sector servicios y su importancia en las Islas Baleares. Las actividades económicas y los sectores productivos de las Islas Baleares, de España y de Europa.

Tabla 1. Cuadro-resumen del currículo de Ciencias Sociales de Educación Primaria relativo a Geografía en la Comunidad Autónoma de las Islas Baleares

Fuente: Elaboración propia a partir de http://weib.caib.es/Normativa/Curriculum_IB/educacio_primaria_lomqe_htm

Los contenidos están organizados, de acuerdo con el juego de escalas, de lo cercano a lo lejano. Se sigue así una compartimentación espacial respetando de forma estricta los principios de Piaget (Bale, 1989). Por tanto, los contenidos geográficos se organizan de forma concéntrica, desde la gran escala (el hogar, la escuela) hacia escalas más pequeñas. El documento que regula dicho currículo apuesta de forma explícita por esa forma de organización:

“...Es muy importante en la educación primaria que el aprendizaje tenga siempre como punto de partida el entorno próximo de los alumnos y a partir de este punto puedan ir asumiendo contenidos cada vez más lejanos, tanto en el espacio como en el tiempo..." (GIB, 2014). 
Desde ese punto de vista, es lógico que sólo se haga referencia a las Islas Baleares, España y Europa, como espacios geográficos concéntricos a los que el alumnado pertenece. Los contenidos del currículo de Ciencias Sociales en Educación Primaria presentan una organización concéntrica (Nadal, 2002: 32), mediante las grandes escalas (el entorno inmediato, la propia comunidad) y abordando en menor medida los órdenes de magnitud superior (Estado y Unión Europea), a través de las escalas medias (España) o pequeñas (Unión Europea). Lo cercano y lo lejano organizan los contenidos del currículo de Ciencias Sociales del alumno de Primaria de esta comunidad. En un mundo globalizado, han aparecido reflexiones que discuten esa forma de organización de los contenidos, en comparación a otras propuestas, destacando la labor desarrollada en el Reino Unido (Nadal, 2002: 33). En su día, Horacio Capel y Luis Urteaga (1986: 8) afirmaban que "... la sumisión al mandato de lo cercano supone una empobrecedora reducción de los contenidos y temas de estudio..." y que, en todo caso, puede ser una forma válida “...en los cursos iniciales de la enseñanza primaria...", aunque “...entraña serios problemas en la segunda etapa de la EGB y en la enseñanza media...". Y todo eso debido a que "los procesos locales, regionales o nacionales sólo pueden ser satisfactoriamente explicados insertándolos en un ámbito global".

De todo ello se infiere que los contenidos que integra el currículo vigente son los que deben conocer los alumnos universitarios de los estudios de grado en Educación Primaria. Por otro lado, la enseñanza de la Geografía continúa durante la enseñanza secundaria obligatoria (ESO) y, además, buena parte del alumnado ha cursado una asignatura de Geografía de España durante el Bachillerato.

\section{Metodología: una prueba para analizar los conocimientos mínimos de Geografía}

La Prueba Objetiva de conocimientos mínimos se ha elaborado a partir de la consulta de los contenidos de Geografía en el Currículo de Educación Primaria. Sus resultados nos han permitido evaluar hasta qué punto los alumnos los han interiorizado tras su dilatada trayectoria académica.

Desde nuestra hipótesis de partida, el alumnado de segundo curso del grado de Educación Primaria debería tener dichos fundamentos, por su carácter básico y por formar parte de los conocimientos mínimos exigibles. La prueba diseñada constó de 20 preguntas (tabla 2). En la primera cuestión se les preguntaba cuál había sido la última vez que habían cursado una materia exclusiva o parcialmente de Geografía con el objeto de analizar si la presencia -escasa desde siempre- de asignaturas de Geografía en el organigrama de materias de la enseñanza primaria y secundaria podía ayudar a interpretar los resultados. A partir de ahí, las cuestiones planteadas fueron las siguientes: 
1 Señala cuál fue el último curso académico en el que cursaste contenidos de Geografía en materia más amplia (Conocimiento del Medio, Ciencias Sociales) o una asignatura completa de Geografía

$2 \quad$ ¿Qué tipos de clima hay en la Península Ibérica?

3 Una tasa de fecundidad general de 2,3 ¿qué significa?

$4 \quad$ ¿De qué trata el informe Meadows?

$5 \quad$ ¿Qué es el sector quinario?

$6 \quad$ ¿Cuáles son los municipios de Baleares de menor extensión y de menor población?

$7 \quad$ ¿A qué se refieren las teorías maltusianas?

$8 \quad$ ¿Cuál es la cota de mayor altitud de Eivissa?

9 ¿Qué isla es la más seca de las Baleares?

10 ¿En qué zona de la isla de Mallorca el régimen pluviométrico es más intenso?

11 ¿Qué son y de qué nos informan los meridianos?

12 ¿A qué tipo de mapa corresponde el que se adjunta?

13 ¿Qué definía el ciclo demográfico antiguo?

14 ¿Cuándo nació la Unión Europea?

15 ¿Qué crean en los polos las células convectivas de la dinámica general atmosférica?

16 ¿De qué es una prolongación marina el Promontorio Balear?

17 ¿Cuál es actualmente el segundo municipio más poblado de Mallorca?

18 ¿A qué comunidad vegetal pertenece el acebuche?

19 ¿Qué es el Camp d'en Torrella en Santanyí (depresión exocárstica)?

20 ¿Qué sierras o cordilleras limitan con la meseta central de la península?

Tabla 2. Cuestiones de la prueba piloto

Fuente: Elaboración propia

En resumen, y en relación a las temáticas, 9 preguntas corresponden a Geografía Física (y de éstas, 4 se refieren a la Climatología, 4 a la Geomorfología y 1 a la Biogeografía); y 8 a Geografía Humana (5 cuestiones son del ámbito de estudio de la Geodemografía, 1 sobre actividades económicas y 1 sobre organización territorial). Finalmente, desde el punto de vista del ámbito territorial, 6 preguntas tratan sobre las Islas Baleares, 3 sobre España y 1 sobre Europa. Las restantes son preguntas genéricas, de carácter conceptual y se refieren en todo momento a los contenidos propios de Ciencias Sociales para Educación Primaria.

\section{Resultados}

\section{El alumnado no posee los conocimientos mínimos}

Los resultados de la prueba objetiva (tabla 3 ) ponen de manifiesto que el alumnado no alcanza los conocimientos mínimos exigibles. Las puntuaciones son muy bajas y nos obligan a plantear seriamente la pregunta: ¿Es posible enseñar Geografía sin saber Geografía?

En segundo curso del grado de Educación Primaria, para el curso académico 2014/15, había en conjunto 199 alumnos. Realizaron la prueba 171 alumnos, un 85,9\% 
del total, y sólo la superaron 55 (32,2\%). En consecuencia, suspendieron 116 alumnos, una abrumante mayoría, que representa un $68,8 \%$ del conjunto de participantes.

\begin{tabular}{ccc}
\hline Calificaciones & $\begin{array}{c}\text { Número de } \\
\text { alumnos }\end{array}$ & $\%$ \\
\hline Inferior a 4 & 116 & $68 \%$ \\
\hline Entre 5 y 6,9 & 48 & $28 \%$ \\
\hline Superior a 7 & 7 & $4 \%$ \\
\hline Total & 171 & $100 \%$
\end{tabular}

Tabla 3. Calificaciones obtenidas en la prueba de conocimientos mínimos

Fuente: Elaboración propia a partir de las pruebas realizadas

La gráfica 1 describe la distribución de los aciertos en las cuestiones planteadas. De las 19 preguntas de contenido, sólo en 7 de ellas el número de respuestas correctas es superior a la mitad de las pruebas realizadas. Se trata de las preguntas: ¿Qué tipos de clima hay en la Península Ibérica? (150 respuestas correctas), ¿Qué sierras o cordilleras limitan con la meseta central de la península? (130 respuestas correctas), ¿Cuándo nació la Unión Europea? (113 respuestas correctas), ¿Qué crean en los polos las células convectivas de la dinámica general atmosférica? (102 respuestas correctas), ¿Qué municipios de Baleares son los de menor extensión y de menor población? (98 respuestas correctas), ¿Qué área es la más pluviosa de Mallorca? (98 respuestas correctas) y ¿Qué isla es la más seca de las Baleares? (91 respuestas correctas). De ese grupo, 5 corresponden a Geografía Física y 2 a Geografía Humana, mientras que 3 se refieren al entorno propio (islas Baleares), 2 al medio inmediato (España) y 1 a los espacios más alejados (Europa).

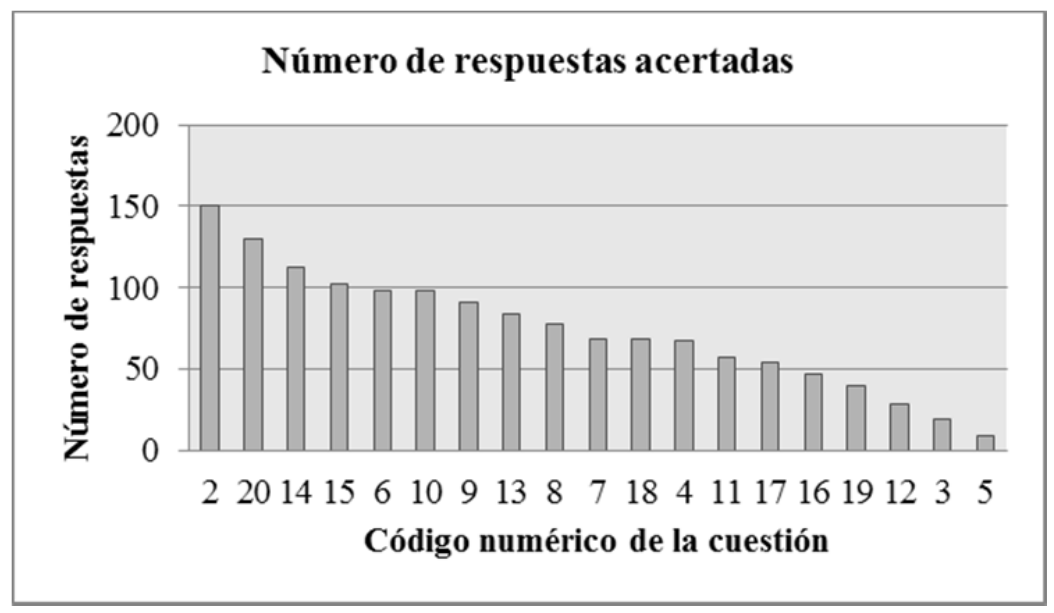

Gráfica 1. Distribución de respuestas acertadas en la prueba objetiva Fuente: Elaboración propia a partir de las pruebas realizadas 
Por otra parte, hay un conjunto de 4 cuestiones a las que respondieron correctamente un porcentaje de encuestados inferior al 25\%: ¿Qué es el sector quinario? (9 respuestas correctas), Una tasa de fecundidad general de 2,3 ¿qué significa? (19 respuestas correctas), ¿A qué tipo de mapa corresponde el que se adjunta? (29 respuestas correctas), ¿Qué es el Camp d'en Torrella en Santanyí? (40 respuestas correctas). Se trata de 3 preguntas de carácter conceptual del ámbito de la Geodemografía y de la Cartografía junto con otra referida a un tipo de forma propia de modelado cárstico en una zona de Mallorca. Finalmente, queda un grupo de 8 preguntas a las que sólo responden correctamente entre el $25 \%$ y el $50 \%$ de alumnos.

La media de las puntuaciones obtenidas es de un 4,35, con un promedio de 8,23 respuestas correctas. El análisis estadístico pone de manifiesto que existe un grupo importante situado en la frontera del $50 \%$ de aciertos. De hecho, hasta un total de 49 alumnos (29\%) tienen una puntuación superior a 4 e inferior a 5. Dicho colectivo, con uno o dos aciertos más, hubiera hecho crecer el total de participantes que han superado la prueba hasta un $61 \%$. No obstante, no debemos de olvidar que sólo 7 alumnos tienen una puntación de notable, sin ningún Sobresaliente, y que la máxima nota es un 8,42. La mayoría de aprobados tiene una puntación mínima que oscila entre el 5 y el 6 (35 alumnos) y sólo 13 de ellos tienen una nota que oscila entre el 6 y el 7.

Además de la nota obtenida en la prueba objetiva, contamos con información sobre varios parámetros estadísticos referidos a la trayectoria académica y vital del alumnado, usada en la investigación sobre la escasa asimilación de los contenidos de Geografía. Estos indicadores son: la presencia de la Geografía en su formación, la edad y el sexo, la nota de la Prueba de Acceso a la Universidad, la nota media de su expediente académico, el tipo de acceso a la Universidad y el nivel de estudios de sus progenitores. A este último aspecto sólo respondieron 105 (61,4\%).

Correlaciones entre variables: no existe relación entre edad y trayectoria con los conocimientos mínimos en Geografia

La nota obtenida de la prueba, como dato cuantitativo, puede correlacionarse con algunos de los parámetros o indicadores a los que nos hemos referido antes, para así poder iniciar el análisis de las variables explicativas del fracaso obtenido. Para ello elaboramos la tabla 4 con los resultados de las correlaciones de Pearson (correlación lineal), donde hemos correlacionado los resultados del examen con algunos indicadores estadísticos cuantitativos de carácter demográfico (edad), académico (nota media de acceso a la selectividad, nota media del expediente) e intrínseco a la prueba misma (número total de respuestas realizadas). 


\begin{tabular}{ll}
\hline Edad & $-0,125012$ \\
\hline Nota Prueba Acceso & 0,3151316 \\
\hline Nota media expediente & 0,3165903 \\
\hline $\begin{array}{l}\text { Total de cuestiones } \\
\text { respondidas }\end{array}$ & 0,6014218 \\
\hline
\end{tabular}

Tabla 4. Correlaciones de Pearson de las notas de la prueba piloto Fuente: Elaboración propia a partir de los resultados de la prueba

La tabla 4 con los resultados de las correlaciones de Pearson entre la nota obtenida en la prueba y las variables utilizadas muestra que ni la edad, ni su trayectoria académica (nota de acceso, media del expediente) son explicativas de los conocimientos mínimos en Geografía, al tener un escaso grado de significación. Por otra parte, la correlación es significativa en relación a la cantidad de cuestiones respondidas. Ello pone de manifiesto que el fracaso detectado responde a una gran cantidad de cuestiones sin respuesta. Teniendo en cuenta que los errores no tenían penalización alguna en la nota final, nos induce a pensar que no respondieron por un desconocimiento absoluto sobre lo que se preguntaba.

\section{La presencia de la Geografía en la trayectoria académica del alumnado}

La presencia de la Geografía en el currículo es otro aspecto muy importante sobre el que recapacitar. En este sentido, discriminamos los alumnos que habían cursado Geografía de España en segundo curso de Bachillerato de aquéllos que habían dejado la Geografía en las materias de Ciencias Sociales de ESO. No sólo los primeros eran mayoría (110 alumnos) frente a los que sólo habían cursado contenidos de Geografía hasta ESO (61 alumnos), sino que además obtienen mejores resultados. Los que cursaron Geografía de España en segundo de Bachillerato presentan un porcentaje de aprobados del $42 \%$, frente al $31,1 \%$ de los que no cursaron contenidos de Geografía desde ESO.

Resulta evidente el escaso peso de la Geografía en el diseño de contenidos del sistema de enseñanza primaria y secundaria en España. Hace tiempo que la Geografía ha retrocedido en el sistema educativo español. En su momento, Capel y Urteaga (1986: 25) ya manifestaban su preocupación ante la pérdida de identidad (inmersos sus contenidos, como ahora, en las materias Ciencias Sociales y Ciencias Naturales) y el retroceso relativo de la Geografía en la EGB y también en el BUP. Esa pérdida de presencia se ha mantenido desde entonces en la enseñanza primaria así como también su escaso peso en el Bachillerato. En cambio, en otros países, la problemática que plantea la enseñanza de la Geografía es distinta. En Holanda, la Geografía es una materia independiente en casi toda la educación primaria y también en la secundaria. Los alumnos de entre 10 y 15 años de edad reciben entre 1 y 1,5 horas de Geografía semanales. En la actualidad, se reclama, por otra parte, una enseñanza de la Geografía que haga hincapié en estructuras conceptuales más que en los procedimientos y se reivindica un mayor énfasis en bases geográficas, que el alumno precisa para buscar su 
propio camino en un mundo que cambia a gran velocidad (Van der Schee; Van der Vaart, 2005: 81-82).

Asimismo resulta poco significativo el peso de la Geografía en los actuales estudios universitarios de formación de futuros maestros. No es una cuestión baladí y también genera debate en otras latitudes y entre sus respectivos colectivos de geógrafos. En Holanda, el tiempo dedicado a la Geografía en la formación del currículo del docente de Educación Primaria es bastante limitado y sometido a la presión de las encuestas escolares. Parece ser que la Geografía es la materia peor enseñada en las escuelas de educación primaria holandesas (Van der Schee; Van der Vaart, 2005: 80). En nuestro contexto, y en relación a la formación de los futuros maestros, no nos sorprende que en el último Anuari de l'Educació de les Illes Balears se afirme que:

"Las didácticas especificas... no encuentran el encaje adecuado en los actuales planes de estudios, ya que la carga docente que soportan es insuficiente para garantizar una formación adecuada en relación a estos contenidos" (Vidal; Oliver, 2014: 168).

Menor presencia tienen aún las materias o asignaturas de contenidos, algo que desde nuestro punto de vista falta en casi todos los planes de los estudios de Maestro en España. En realidad, no se da un equilibrio entre ambos subsistemas, la disciplinar y la formativa:

“...en el actual plan de estudios de Magisterio hay una abultada presencia de materias psicopedagógicas y educativas, a nuestro entender excesiva y atomizada, con una ausencia total, en la mayoría de universidades, de contenidos disciplinares de cualquier tipo, imprescindibles para el futuro maestro/a..." (García Ruiz, 2003: 9).

Por otra parte, Sureda, en su estudio crítico sobre la formación de los futuros maestros, subraya la preocupación que esa problemática suscita en el Gobierno de la Comunidad de Madrid, que ha suscrito un convenio de colaboración con las universidades madrileñas, públicas y privadas, para que éstas velen por una reestructuración de los planes de estudios. Esa readecuación ha de rebajar la parte pedagógica y didáctica y elevar el peso de los fundamentos. Además, las universidades madrileñas se comprometieron a realizar una prueba de acceso independiente a la PAU, con el objetivo de asegurar una preparación mínima previa de los futuros maestros (Sureda, 2014: 57).

La convicción sobre la importancia de la Geografía en los estudios de Educación Primaria traspasa nuestras fronteras. Cristiano Giorda y Maria Teresa di Palma (2011) resumen, en un interesante trabajo, la labor llevada a cabo por un grupo de 22 geógrafos reunidos en un denominado Grupo de Coordinación de la Geografía, creado para generar un debate en la red sobre el papel que debe de jugar la Geografía en la enseñanza primaria italiana y la formación de maestros. Dicho grupo nace en el contexto de la reforma de la enseñanza obligatoria llevada a cabo en 2007 y tras el Decreto-Ley 249/2010, que reguló los nuevos planes de estudio de maestro, cuya duración es, desde entonces, de 5 años.

Giorda y di Palma (2011: 188) manifiestan su convicción del gran valor educativo de la Geografía por su carácter interdisciplinario, muy indicado para abordar temas en 
un sistema didáctico integrado. Por otra parte, pretenden romper con el enciclopedismo dogmático que se desprende del documento ministerial postulándose a favor de una Geografía dinámica, que aborde la cuestión medioambiental como el resultado de la interacción entre sistemas naturales, socio-culturales, económicos y políticos, que focalice en aspectos educacionales como la sostenibilidad, la interculturalidad y la diversidad. En definitiva, el Grupo de Coordinación de la Geografía reivindica un giro en la óptica que se deben dar a los contenidos de la Geografía en el nuevo grado de 5 años de Educación Primaria en Italia y donde la Geografía juega un destacado papel. De hecho, el documento ha abierto el debate sobre la importancia de la Geografía en la Educación con la pretensión de intentar dotar de un currículo homogéneo a los futuros maestros italianos de primaria (Giorda, di Palma, 2011: 190).

En España, es evidente la falta de coherencia interna que se detecta analizando los planes de estudios de las Universidades que participaron en la elaboración del Libro Blanco de los estudios de Maestro. Este documento marcó unas directrices laxas, que cada universidad interpretó lanzando su propia propuesta. A partir de las consultas efectuadas en las páginas web de las diversas universidades españolas en las que se imparten estudios de grado de Educación Primaria se deduce, con claridad meridiana, que la Geografía tiene un escaso peso en los estudios de Maestro. De hecho, son muy pocas las que ofrecen asignaturas temáticas de Geografía y, en su mayoría, se trata de asignaturas optativas de menciones e itinerarios relacionados con las Ciencias Sociales. Ocurre esto en las Universidades de Valladolid (sedes de Soria y Palencia con Geografía de Europa, y sede de Valladolid con Geografía de los Paisajes), Alcalá de Henares (Geografía General), Oviedo (Fundamentos de Geografía), Sevilla (Fundamentos de Geografía), La Laguna (Geografía de Canarias I y II) y Lleida (Geografía e Historia de Cataluña). También se construyen currículos con asignaturas donde comparten contenidos viejos aliados académicos: Geografía e Historia; Geografía, Historia e Historia del Arte e incluso Geografía, Historia y Filosofía, tal como pudimos constatar en las guías docentes de 8 asignaturas.

Otras veces, la Geografía como sujeto se inserta en una asignatura donde los contenidos geográficos (saber Geografia) comparten protagonismo con los fundamentos didácticos de la materia (cómo enseñar Geografía). En algunas ocasiones, esa duplicidad de planteamientos se explicita en el mismo nombre de la asignatura (Fundamentos y Didáctica de la Geografía, en la Universidad Complutense de Madrid). Paralelamente, existen casos donde esa dualidad no se explicita en el nombre de la asignatura, que es claramente de vocación didáctica (Didáctica de las Ciencias Sociales: Geografía, Didáctica de la Geografía, Ciencias Sociales: Geografía y su Didáctica o simplemente Didáctica de las Ciencias Sociales). Esa dualidad de objetivos se observa en 13 de las 78 asignaturas analizadas, aunque son mayoría (49 asignaturas) las materias concebidas únicamente bajo el prisma de la Didáctica. En este grupo los planteamientos son también muy diferenciados: los que conciben la materia como una Didáctica específica de Geografía dirigida a futuros maestros (Didáctica de la Geografía, en la Universidad de Barcelona, o Didáctica de las Ciencias Sociales: Geografía, en la Universitat d'Alacant); o planteamientos didácticos, de gran carga teórica, donde la Geografía queda diluida en una propuesta integral de las Ciencias Sociales (caso Cultura, medio y enseñanza de lo Social, en la Universidad de 
Cantabria). En resumen, las asignaturas con planteamientos didácticos (cómo enseñar) tienen un mayor peso que las asignaturas de contenidos, lo que sin lugar a dudas nos reafirma en la orfandad de la Geografía en el sistema educativo español que repercute en la formación de los futuros maestros. Por ello, no es descabellada la afirmación según la cual "...los estudiantes, que no hayan hecho el Bachillerato de Humanidades, llegarán a la escuela sin haber estudiado más Geografía que la incluida en el área de Ciencias Sociales de la ESO..."(García Ruiz, 2003: 8).

\section{La incidencia de los factores demográficos en los conocimientos mínimos de} Geografía entre los estudiantes de Educación Primaria

\section{La edad}

Desde esta variable, el fracaso es mayor entre los de mayor edad biológica (tabla 5). Esto contraviene las tesis apuntadas por Gustavo Nieto Barbero (2013) en un trabajo reciente con finalidades muy similares al nuestro. Según dicho autor, la puntuación obtenida es mayor entre los de mayor edad, hecho que corroboraría la experiencia como un factor clave en el aprendizaje geográfico, incluso más que la escuela. Aun así, la muestra sólo incluye edades entre 20 y 25 años y la correlación de Pearson es baja $\left(0,2\right.$ de $\left.\mathrm{R}^{2}\right)$. Los avances del autor ahondarían en la idea de que la renovación educativa en relación a la Geografía no ha producido los efectos deseados y que, por tanto, se continúa enseñando de forma muy tradicional porque los contenidos no son significativos y la metodología seguida no permite asimilarlos.

En nuestro caso, la edad no es causa explicativa de los resultados obtenidos (la correlación entre ambas variables es de $\mathrm{R}^{2}=-0,12$, un resultado que indica nula relación). En este sentido, la mayoría de los que realizaron la prueba nacieron en 1995, año de promoción que corresponde al cursar segundo curso de los estudios de Maestro y que accedieron a la universidad tras el curso 2012/13 sin haber perdido ningún curso en su trayectoria académica, y son los que obtienen mejores resultados. Un $32,9 \%$ de ellos superaron la prueba de conocimientos mínimos en Geografía, mientras que sólo un $28,6 \%$ de los nacidos entre 1991 y 1994, ambos inclusive (entre 21 y 23 años), tuvieron un resultado positivo. Y aún mayor es el porcentaje del fracaso entre los de más 23 años (nacidos durante o antes de 1990), con un porcentaje de aprobados del $17 \%$.

\begin{tabular}{lccccc}
\hline & Inferior a 4 & Entre 5 y 6,9 & Superior a 7 & Total & $\begin{array}{c}\text { \% de } \\
\text { aprobados }\end{array}$ \\
\hline Antes de 1990 & 29 & 6 & 0 & 35 & $17 \%$ \\
\hline $\begin{array}{l}\text { Entre 1991 y } \\
\text { 1994 }\end{array}$ & 41 & 18 & 4 & 63 & $29 \%$ \\
\hline En 1995 & 46 & 24 & 3 & 73 & $33 \%$ \\
\hline
\end{tabular}

Tabla 5. Resultados de la prueba según año de nacimiento

Fuente: Elaboración propia a partir de los resultados de la prueba y las variables personales del alumnado

Los más jóvenes son los que obtienen mejores resultados $\mathrm{y}$, por tanto, los que mejor han interiorizado los conocimientos académicos de Geografía en su trayectoria. La edad, la experiencia acumulada, no ha jugado a favor de los mayores sino más bien al 
contrario, pues los porcentajes de fracaso fueron superiores entre el grupo que había nacido antes de 1990 y los que lo hicieron entre 1991 y 1994.

\section{El género}

En la tabla 6 se observa que a pesar de ser unos estudios con una significativa mayoría del sexo femenino (realizaron la prueba 127 mujeres y 44 varones), son éstos los que obtienen mejores resultados en la prueba de conocimientos mínimos en Geografía, superando la prueba un $43,2 \%$ frente a sólo un $28,3 \%$ de las mujeres.

\begin{tabular}{lcccc}
\hline & $\begin{array}{c}\text { Nota } \\
\text { inferior a 5 }\end{array}$ & $\begin{array}{c}\text { Nota } \\
\text { superior a 5 }\end{array}$ & Total & $\begin{array}{c}\text { \% superado } \\
\text { la prueba }\end{array}$ \\
\hline Hombres & 25 & 19 & 44 & $43 \%$ \\
\hline Mujeres & 91 & 36 & 127 & $28 \%$ \\
\hline
\end{tabular}

Tabla 6. Resultados de la prueba según sexo

Fuente: Elaboración propia a partir de los resultados de la prueba y las variables personales del alumnado

Otros estudios en los que se explotan encuestas cuyo objetivo es analizar los conocimientos mínimos en Geografía (Geography literacy o también Place Location Knowlege (PLK)), resaltan las diferencias en relación al género. Whinship (2004: 62), en cuya muestra se encuentran alumnos de la Universidad de Virginia, llega a la evidencia empírica de que el $75 \%$ de los hombres superan la media frente al $45 \%$ de las féminas. Teoriza que esas diferencias se explican a partir de la teoría de la diferencia, según la cual variables socioculturales explicarían esas diferencias en la aptitud ante los conocimientos geográficos de ambos sexos (exposición a la Geografía, precocidad en la formación y conocimiento espacial, expectativas familiares) (Whinship, 2004: 30 ). En otro estudio, donde el mapa mental sustituye a la prueba objetiva o encuesta, Nishimoto (2012: 23) demuestra que los chicos conocen más países por continentes que las chicas. En nuestro caso, sólo hemos podido relacionar el género con los resultados de la prueba y no con otros parámetros indicativos de usos y costumbres de una experiencia vital más "geográfica".

La repercusión de los condicionantes académicos y socioculturales en los conocimientos mínimos de Geografia entre los estudiantes de Educación Primaria

La edad "académica" tampoco influye de forma significativa en los resultados obtenidos. La tabla 7 muestra como los que realizaron la prueba de acceso a la Universidad hasta el curso 2004/05 obtienen un resultado negativo en un 77,8\% de los casos. Los que la realizaron entre el curso 2005/06 y 2009/10 suspenden la prueba de conocimientos mínimos en un $73,3 \%$, porcentaje que desciende hasta el $66,7 \%$ entre los que accedieron a la Universidad desde el curso 2010/11. 


\begin{tabular}{lccc}
\hline & $\begin{array}{c}\text { Nota } \\
\text { inferior a } 5\end{array}$ & Total & $\%$ \\
\hline Antes del curso 2004/2005 & 7 & 9 & $77,8 \%$ \\
\hline $\begin{array}{l}\text { Entre el curso 2005/06 y el } \\
\text { curso 2009/10 }\end{array}$ & 11 & 15 & $73,3 \%$ \\
\hline A partir del curso 2010/11 & 98 & 147 & $66,7 \%$ \\
\hline
\end{tabular}

Tabla 7. Resultados de la prueba según el curso de acceso a la Universidad

Fuente: Elaboración propia a partir de los resultados de la prueba y las variables personales del alumnado

Respecto al sistema de acceso, no es clarificador con los alumnos que accedieron a la universidad hace ya unos años. La tabla 8 evidencia que los que obtienen mejores resultados son los que realizaron la prueba de acceso a Grado, con un $35 \%$ de aprobados. Tras ellos se sitúan los que provienen de un módulo profesional, con un $33,3 \%$ de aprobados. En tercer lugar, aun siendo un grupo anecdótico, se hallan los que ya tienen un título universitario, grupo que obtiene un $25 \%$ de aprobados. En los últimos peldaños se sitúan los alumnos que realizaron la prueba de acceso de mayores de 25 años, con un 16,6\% de aprobados y los que las realizaron antes de 2010, ya fuera desde el Bachillerato de la LOGSE o desde COU, con sólo un 13,3\% de aprobados.

\begin{tabular}{|c|c|c|c|c|}
\hline & $\begin{array}{c}\text { Nota } \\
\text { inferior a } \\
5 \\
\end{array}$ & $\begin{array}{c}\text { Nota } \\
\text { superio } \\
\text { r a } 5\end{array}$ & $\begin{array}{c}\text { Tota } \\
\text { I }\end{array}$ & $\begin{array}{c}\% \\
\text { superado } \\
\text { la prueba }\end{array}$ \\
\hline $\begin{array}{l}\text { Acceso por cambio de universidad/estudios } \\
\text { universitarios oficiales españoles }\end{array}$ & 1 & 0 & 1 & $0 \%$ \\
\hline Formación profesional (Título de técnico superior) & 12 & 6 & 18 & $33 \%$ \\
\hline $\begin{array}{l}\text { Mayores de } 25 \text { años (Pruebas de acceso a mayores } \\
\text { de } 25 \text { años) }\end{array}$ & 5 & 1 & 6 & $17 \%$ \\
\hline $\begin{array}{l}\text { Pruebas de acceso a la universidad (Bachillerato } \\
\text { (LOGSE) con PAU) }\end{array}$ & 13 & 2 & 15 & $13 \%$ \\
\hline $\begin{array}{l}\text { Pruebas de acceso a la universidad (prueba de } \\
\text { acceso a Grado) }\end{array}$ & 82 & 44 & 126 & $35 \%$ \\
\hline $\begin{array}{lllll}\begin{array}{l}\text { Pruebas de acceso a la universidad (sistema } \\
\text { educativo extranjero) }\end{array} & & & \\
\end{array}$ & 0 & 1 & 1 & $100 \%$ \\
\hline Título, diplomatura o primer ciclo (licenciatura) & 3 & 1 & 4 & $25 \%$ \\
\hline Total general & 116 & 55 & 171 & $32 \%$ \\
\hline
\end{tabular}

Tabla 8. Resultados de la prueba según forma de acceso a la Universidad

Fuente: Elaboración propia a partir de los resultados de la prueba y las variables personales del alumnado 
La nota de acceso y la nota media tampoco fueron variables claramente explicativas de los resultados de la prueba (las correlaciones de Pearson de ambas variables, $\mathrm{R}^{2}=0,31$ y $\mathrm{R}^{2}=0,32$, respectivamente, no resultan significativas al respecto). Es decir, la asimilación o interiorización de conocimientos en Geografía no responde de forma determinante al bagaje en conocimientos, habilidades y capacidades del estudiantado, ni tampoco tiene mucho que ver con su trayectoria académica, como refleja la media del expediente.

Otro aspecto a valorar es el ambiente en el que se han educado. Para ello, hemos considerado el nivel de estudios de los padres como un indicador de su origen social y de si se han formado en un entorno familiar estimulante.

\begin{tabular}{lccc}
\hline & $\begin{array}{c}\text { A: Nota superior } \\
\text { a 5 }\end{array}$ & $\begin{array}{c}\text { B: Nota } \\
\text { inferior a 5 }\end{array}$ & A/B \\
\hline Nivel bajo & 3 & 4 & 0,75 \\
\hline Nivel básico & 11 & 25 & 0,44 \\
\hline Nivel medio & 16 & 28 & 0,57 \\
\hline Nivel superior & 10 & 8 & 1,25 \\
\hline Total general & 40 & 65 & 0,62 \\
\hline
\end{tabular}

Tabla 9. Resultados de la prueba según nivel de estudios de los progenitores

Fuente: Elaboración propia a partir de los resultados de la prueba y las variables personales del alumnado

La tabla 9 revela los resultados sobre el total de los 105 alumnos que nos informaron del nivel de estudios de sus padres, cifra que representa un $61,4 \%$ del total, obteniendo resultados significativos al respecto. Los participantes con padres con nivel de estudios superiores (ambos cónyuges con título universitario) es el único segmento donde los aprobados superan a los suspendidos, con una relación de 1,25. Algo inferior es la conseguida en el segmento cuyos padres han sido adscritos al nivel medio (ambos con bachillerato o FP, o también bachillerato y COU, o uno de los dos con título universitario y el otro con bachillerato o FP), siendo ya mayor en este grupo el número de suspendidos al de aprobados (relación de 0,57). Se incrementa todavía más el desequilibrio en el grupo cuyos padres tienen un nivel básico de estudios (ambos con estudios primarios, o con uno de los dos con estudios de bachillerato o similar), con una relación entre aprobados y suspendidos de 0,44 . Únicamente rompe mínimamente esta tendencia descendente el grupo inferior, el de los alumnos con unos padres con un nivel de estudios bajo (ambos sin estudios o uno de los dos con estudios primarios), siendo en este caso la relación entre aprobados y suspendidos de 0,75 , aunque debemos de relativizar ese aparente ruptura con la línea descendente por el escaso peso absoluto de dicho grupo ( 7 en total). 


\section{Conclusiones y discusión}

Ante el evidente fracaso en los resultados de la prueba objetiva, cabe plantearse las siguientes cuestiones:

a. ¿Es la escasa presencia de la Geografía en los currículos de la enseñanza primaria y secundaria, una causa explicativa de los resultados obtenidos?

b. ¿Existen variables académicas, demográficas y sociales que nos ayuden, ni siquiera parcialmente, a encontrar explicaciones a la escasa interiorización de contenidos geográficos mínimos entre los estudiantes del grado de Maestro?

La prueba piloto realizada ha puesto de manifiesto:

1. Que los futuros maestros no han asimilado los conocimientos geográficos mínimos para impartir con solvencia las materias que incluyen esta disciplina (actualmente Ciencias Sociales, según la LOMCE).

2. No existe ninguna variable con un peso determinante para explicar el escaso bagaje en Geografía de los futuros maestros.

3. La presencia de asignaturas y contenidos de Geografía es muy escasa en el sistema educativo español desde hace ya mucho tiempo (Ley General de Educación de 1970), lo que sin duda contribuye a la escasa formación geográfica de los futuros profesionales de la enseñanza.

4. Los alumnos pertenecientes a familias con un mayor nivel de estudios $y$, por tanto, con un entorno estimulante, obtienen mejores resultados.

5. Existe una preocupación manifiesta por esta cuestión en otros países europeos. Tanto en Italia como en Holanda, los trabajos analizados enfatizan en la necesidad de aumentar las materias de Geografía en el sistema educativo y también en la formación de futuros profesionales docentes de Educación Primaria.

6. Las universidades españolas, desde la consolidación de los estudios de grado (curso académico 2010/11), han optado preferentemente por asignaturas de perfil psicopedagógico frente a asignaturas de fundamentos -entre ellas, la Geografía-, en los estudios de Maestro en Educación Primaria.

7. Otras materias del plan de estudios de Maestro, como Matemáticas, han hecho hincapié en la problemática de los conocimientos mínimos, aun teniendo una presencia mucho mayor tanto en los planes de estudio de Maestro como en el conjunto del sistema educativo español.

8. La futura remodelación de los planes de estudio del grado en Educación Primaria deberá hacer énfasis en esta cuestión para evitar que los graduados de Educación Primaria accedan al ámbito profesional con importantes carencias en los conocimientos mínimos sobre las materias que deben enseñar. 


\section{Referencias bibliográficas}

AGENCIA NACIONAL DE EVALUACIÓN DE LA CALIDAD Y ACREDITACIÓN (ANECA) (2005). Libro Blanco. Título de Grado en Magisterio. Volumen 1. En: http://www.aneca.es/var/media/150404/libroblanco_jun05_magisterio1.pdf octubre 2014

BALE, J. (1989). Didáctica de la Geografía en la escuela primaria. Madrid: Ediciones Morata, S.L.

BUZO SÁNCHEZ, I., IBARRA BENLLOCH, P. (2014). La posición de la Geografía en la Educación Secundaria y el Bachillerato. Madrid: AGE. En: http://ciug.cesga.es/PDF/informeage13.pdf, octubre 2014

CAPEL, H., URTEAGA, L. (1986). La Geografía en el currículum de Ciencias Sociales. Geocritica, 61, 5-36.

GARCÍA RUIZ, A. (2003). El conocimiento del medio y su enseñanza práctica en la formación del profesorado de Educación Primaria. Granada: Ed. Nativola.

GIORDA C.; DI PALMA, M.T. (2011). The italian geographers' Document of the University Education of Future Primary School Teachers. Review of International Geographical Education Online (RIGEO), 1(2), 183-196.

GOVERN DE LES ILLES BALEARS. CONSELLERIA D’EDUCACIÓ, CULTURA I UNIVERSITATS (GIB) (2014). Decreto 32/2014, de 18 de julio, por el cual se establece el currículo de Educación Primaria en las Islas Baleares. En: http://weib.caib.es/Normativa/Curriculum_IB/decrets/decret_primaria_32-2014.pdf octubre de 2014

MIRÓ, J.; NADAL, M. (2014). Conocimientos mínimos de la asignatura “Matemàtiques de l'educació primària" Curso 2013-2014. Inédito.

NADAL, I. (2002). Lo cercano y lo lejano como criterio de ordenación de los contenidos del currículo de Ciencias Sociales. Íber. Didáctica de las Ciencias Sociales. Geografia e Historia, 32, 28-40.

NIETO BARBERO, G. (2013). El coneixement de la Geografia acadèmica dels alumnes de grau de mestre en educación primària. En Joan Pagès y Antoni Santisteban (eds): Una mirada al pasado y un proyecto de futuro. Investigación e innovación en didáctica de las ciencias sociales (vol. 2, pp. 489-496). Barcelona: Asociación Universitaria de Profesorado de Didáctica de las Ciencias Sociales.

NISHIMOTO, S. (2012). Evaluating mental mapas. Tesis para la obtención del Grado de Ciencias en Geografía. Universidad de Oregon. En: http://geog.uoregon.edu/edge/EDGE/Projects/2012/S_nishimoto_2012.pdf diciembre 2014.

NORTES, R; NORTES, A. (2014). La competencia matemática en futuros maestros ¿Una realidad o una ficción? Educatio Siglo XXI, 32 (2), 163-194. 
PÉREZ ESTÉVEZ, P.; RAMÍREZ MARTÍNEZ, S.; XOUTO GONZÁLEZ, X. (1997). El área de conocimiento del medio ¿Un cajón de sastre? Investigación en la escuela, 31, 17-40.

SOCAS, M.M. (2011). Aprendizaje y enseñanza de las matemáticas en Educación Primaria. Buenas pràcticas. Educatio Siglo XXI, 29 (2), 199-224.

SUREDA NEGRE, J. (2014). La formació inicial del profesorat d'infantil i primària. Inédito.

VAN DER SCHEE, J.; VAN DER VAART, R. (2005). Geography Teaching in The Netherlands: Changes and Challenges. International Research in Geographical and Environmental Education, 14:1, 80-82.

VIDAL, J.; OLIVER J.L. (2014). El grau d'Educació Primària de la UIB: passat, present i futur. En Martí X. March i Cerdà (dir.): Anuari de l'Educació de les illes Balears. 2014 (pp. 158-171). Palma: Caixa Colonya. Fundació Guillem Cifre.

WHINSHIP, J.M. (2004). Geographic Literary and world knowledge among undergraduate college students. Tesis para la obtención del Grado de Ciencias en Geografía. Virginia Polytechnic Institute and State University. En: http://scholar.lib.vt.edu/theses/available/etd-11022004144902/unrestricted/Final_Thesis_JWinship.pdf octubre 2014.

\section{Correspondencia con los autores}

Jaume BINIMELIS SEBASTIÁN

Edificio Beatriu de Pinós. Campus Universitario

Carr. de Valldemossa, km 7,5

07122 Palma, Illes Balears

e-mail: jaume.binimelis@uib.es

Antoni ORDINAS GARAU

Edificio Beatriu de Pinós. Campus Universitario

Carr. de Valldemossa, km 7,5, 07122 Palma, Illes Balears

Edificio Beatriu de Pinós. Campus Universitario

Carr. de Valldemossa, km 7,5

07122 Palma, Illes Balears

e-mail: antoni.ordinas@uib.es 